\title{
秸秆燃烧排放 PM 2.5 特征及影响因素研究
}

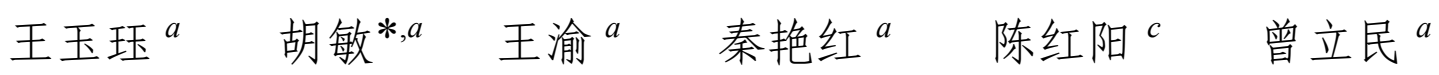 \\ 雷建容 ${ }^{b}$ 黄晓锋 ${ }^{b}$ 何凌燕 $b \quad$ 张瑞芹 ${ }^{c}$ 吴志军 ${ }^{a}$ \\ ( ${ }^{a}$ 环境模拟与污染控制国家重点联合实验室 北京大学环境科学与工程学院 北京 100871) \\ ( ${ }^{b}$ 城市人居环境科学与技术重点实验室 北京大学深圳研究生院环境与能源学院 深圳 518055) \\ ( ${ }^{c}$ 郑州大学化学系 郑州 450001)
}

\begin{abstract}
摘要 生物质燃烧向大气中排放大量颗粒污染物, 在中国, 收获季节大量秸秆被直接露天燃烧处理, 对区域环境质量 和人体健康造成严重影响. 对我国最主要的两种粮食作物玉米和小麦秥秆的露天燃烧进行模拟, 分析颗粒物排放水平, 特征及影响因素. 玉米和小麦秥秆燃烧 $\mathrm{PM}_{2.5}$ 排放因子分别为 1082.8 和 $835.7 \sim 897.3 \mathrm{mg} / \mathrm{kg}$. 有机物是颗粒物最主要组 分, 总量占 $\mathrm{PM}_{2.5}$ 质量的 $42 \% \sim 66 \%$. $\mathrm{Cl}^{-}$和 $\mathrm{K}^{+}$分别占 $\mathrm{PM}_{2.5}$ 总质量的 $4 \% \sim 15 \%$ 和 $2 \% \sim 14 \%, \mathrm{~K}^{+} / \mathrm{EC}$ 值为 $0.5 \sim 3.8$. 各物 种在颗粒物中所占比例与之前研究结果一致. 秸秆含水量和燃烧温度影响 $\mathrm{PM}_{2.5}$ 排放水平和组成特征. 随秥秆含水量增 加, $\mathrm{PM}_{2.5}$ 和 $\mathrm{OC}$ 的排放因子增加; 秸秆含水量增加, 燃烧温度逐渐降低, 由生物质燃料释放进入烟气中的 $\mathrm{K}^{+}$和 $\mathrm{Cl}^{-}$比例逐渐减小导致二者在颗粒物中的比例降低. 我国每年由玉米和小麦秥秆露天燃烧排放的 $\mathrm{PM}_{2.5}$ 和 $\mathrm{OC}$ 分别为 $92.7 \mathrm{Gg}$ 和 $47.5 \mathrm{Gg}$, 在总量中占重要比例.
\end{abstract}

关键词 生物质燃烧; 秸秆; $\mathrm{PM}_{2.5}$; 排放因子; 特征; 影响因素

\section{Characterization and Influence Factors of $\mathrm{PM}_{2.5}$ Emitted from Crop Straw Burning}

\author{
Wang, Yujue ${ }^{a} \quad \mathrm{Hu}, \mathrm{Min}^{*}, a \quad$ Wang, Yu $^{a} \quad$ Qin, Yanhong $^{a} \quad$ Chen, Hongyang ${ }^{c} \quad$ Zeng, Limin ${ }^{a}$ \\ Lei, Jianrong $^{b} \quad$ Huang, Xiaofeng $^{b} \quad$ He, Lingyan $^{b} \quad$ Zhang, Ruiqin $^{c} \quad$ Wu, Zhijun ${ }^{a}$ \\ ( ${ }^{a}$ State Key Joint Laboratory of Environmental Simulation and Pollution Control, College of Environmental \\ Sciences and Engineering, Peking University, Beijing 100871) \\ ( ${ }^{b}$ Key Laboratory for Urban Habitat Environmental Science and Technology, School of Environment and Energy, \\ Peking University Shenzhen Graduate School, Shenzhen 518055) \\ ( ${ }^{c}$ Department of Chemistry, Zhengzhou University, Zhengzhou 450001)
}

Abstract Large quantities of particulate pollutants are emitted into the atmosphere during biomass burning processes. In China, large amounts of agricultural residues are burned in the field during harvest seasons, which influence regional air quality and human health. Corn and wheat are two major crops grown in China, whose burning was simulated in this study. The controlled laboratory simulation of straw burning was performed in the Laboratory of Biomass Burning Simulation at Peking University Shenzhen Graduate School. The burning simulation system was improved and verified. Straw burning aerosols $\left(\mathrm{PM}_{2.5}\right)$ samples were collected and measured by gravimetric method. Organic carbon (OC) and elemental carbon (EC) were measured by thermal/optical method. Water-soluble inorganic ions and organic matter were measured by ion chromatography. Emission level, characterization and influence factors of crop straw burning aerosols are discussed. $\mathrm{PM}_{2.5}$ emission factors of corn and wheat straw burning are 1082.8 and $835.7 \sim 897.3 \mathrm{mg} / \mathrm{kg}$, respectively. Organic matter (OM), which is calculated by multiplying organic carbon (OC) by 1.3 , is the major component of $\mathrm{PM}_{2.5}$, accounting for $42 \% \sim 66 \%$ of the total mass. Nearly half $(37 \% \sim 50 \%)$ of $\mathrm{OM}$ are water soluble. $\mathrm{Cl}^{-}$and $\mathrm{K}^{+}$are two major components among water-soluble inorganic ions, accounting for $4 \% \sim 15 \%$ and $2 \% \sim 14 \%$ of total particle mass, respectively. The $\mathrm{K}^{+} / \mathrm{EC}$ ratio is $0.5 \sim 3.8$. The proportions of these species in $\mathrm{PM}_{2.5}$ are comparable to previous studies. Straw moisture content and burning temperature influence the emission level and characterization of straw burning aerosols. Emission factors of $\mathrm{PM}_{2.5}$ and $\mathrm{OC}$ increase with the increase of straw moisture content because of incomplete burning. With higher moisture content, more thermal energy is used for the evaporation of water, lowering the burning temperature. Then less proportion of $\mathrm{K}^{+}$and $\mathrm{Cl}^{-}$ are released from biomass into the smoke. Therefore, their contributions to the particle mass decrease with the increase of straw moisture content. The emissions of $\mathrm{PM}_{2.5}$ and $\mathrm{OC} / \mathrm{EC}$ by crop straw burning in the field are estimated based on the

*E-mail: minhu@pku.edu.cn; Tel.: 010-62759880

Received January 5, 2016; published February 12, 2016.

Project supported by the Nonprofit Research Projects of Environmental Protection Department of China (No. 201409010), the National Natural Science Foundation of China (Nos. 91544214, 21190052) and the Special Fund for Strategic Pilot Technology Chinese Academy of Sciences (No. XDB05010500). 项目受环保部公益性行业科研专项(No. 201409010)、国家自然科学基金重大项目(Nos. 91544214, 21190052)和中国科学院战略性先导科技专项(No. XDB05010500)资助. 


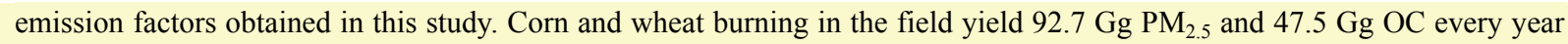
in China, accounting for important fractions among the total mass.

Keywords biomass burning; crop straw; $\mathrm{PM}_{2.5}$; emission factor; characterization; influence factor

\section{1 引言}

生物质燃烧向大气排放大量气态和颗粒态污染物, 影响空气质量和人体健康 ${ }^{[1 \sim 4]}$, 碳质气溶胶是生物质燃 烧排放颗粒物中最主要的组分 ${ }^{[5,6]}$. 1996 2010 年间, 生 物质燃烧对中国有机碳(OC)和元素碳(EC)的贡献分别 为 34\% 45\%和 $63 \% \sim 74 \%$, 且 2000 年之后呈现增加的 趋势 ${ }^{[3]}$. 生物质燃烧既包括森林、木材的燃烧, 也包括农 业废弃物秸秆等的燃烧, 不同种类生物质燃烧排放颗粒 物的水平和特征存在较大差异 ${ }^{[1,6,7]}$. 在中国除木材燃烧 外, 秸秆露天燃烧是生物质燃烧的一类重要形式. 研究 表明，中国每年有 $110 \sim 158 \mathrm{Tg}$, 即 $17 \% \sim 26 \%$ 的农作物 秸秆被直接露天焚烧处理 ${ }^{[8 \sim 10]}$. 中国的生物质燃烧排放 颗粒物源谱特征与木材燃烧为主要生物质燃烧形式的 国家存在差异, 对其秸秆燃烧排放颗粒物的特征进行模 拟和研究具有重要意义.

有机碳是秸秆燃烧排放颗粒物中最主要的组分, 约 占总质量的 $26 \% \sim 85 \%{ }^{[6,11,12]}$. $\mathrm{K}^{+}$作为生物质燃烧示踪 物, 秙秆燃烧过程也有较高浓度的排放 ${ }^{[12 \sim 14]}$. 生物质燃 烧排放颗粒物的特征受多种因素的影响, 燃烧过程中的 环境氧气量、燃料含水量和燃烧温度等是影响燃烧状 态、颗粒物排放水平和特征的重要因素 ${ }^{[15 ~ 17] . ~}$. 目前对生 物质燃烧排放研究主要有实地燃烧实验 $[11,14,18,19]$ 和实验

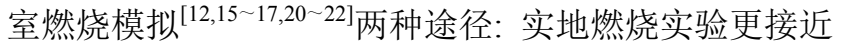
实际秸秆露天燃烧的外界条件, 但实验条件难以控制, 即使是同种秸秆的燃烧排放也可能存在较大差异, 不同 实验结果间的可比性难以评价. 虽然燃烧模拟实验中秸 秆的燃烧量相对实际情况较少, 与实际露天燃烧的环境 条件存在一定差异, 但实验室模拟可以更好控制燃烧条 件和平行性, 是研究生物质燃烧排放影响因素常运用的 方法.

中国作为农业大国, 收获季节存在大量的秸秆集中 露天燃烧现象, 且不同地区和季节温、湿条件不同导致 燃烧状态和排放特征存在差异. 本文选取我国最主要的 两种粮食作物玉米和小麦秸秆, 对其燃烧排放进行模拟 研究, 并讨论秸秆含水量对排放颗粒物水平和特征的影 响. 本研究为我国生物质燃烧排放颗粒物源谱和源解析 工作的完善和推进提供基础数据, 对污染防治措施的制 定具有重要意义.

\section{2 结果与讨论}

\section{$2.1 \mathrm{PM}_{2.5}$ 排放因子}

为比较不同秙秆燃烧排放颗粒物水平的差异, 这一 部分不考虑同种秸秆各含水量梯度间的排放差异, 玉米 秸秆和两地小麦秸秆燃烧排放 $\mathrm{PM}_{2.5}$ 的平均排放因子见
表 1. 玉米秸秆、望都小麦秸秆和郑州小麦秸秆 $\mathrm{PM}_{2.5}$ 的排放因子分别为 $1082.8,835.7$ 和 $897.3 \mathrm{mg} / \mathrm{kg}$. Hays 等 ${ }^{[12]}(2005)$ 对秸秆燃烧排放的模拟结果显示, 小麦和水 稻秙秆 $\mathrm{PM}_{2.5}$ 排放因子分别为 4710 和 $12950 \mathrm{mg} / \mathrm{kg} ; \mathrm{Li}$ 等 ${ }^{[11]}$ (2007)研究结果表明小麦和玉米秸秆燃烧 $\mathrm{PM}_{2.5}$ 排 放因子分别为 7600 和 $11700 \mathrm{mg} / \mathrm{kg}$; Andreae 等 ${ }^{[6]}(2001)$ 得到农业废弃物燃烧 $\mathrm{PM}_{2.5}$ 排放因子为 $3900 \mathrm{mg} / \mathrm{kg}$. 与 之相比, 本研究得到的排放因子较低, 这与不同研究中 的燃料种类及其含水量、模拟方式和燃烧条件等因素有 关. 两地小麦秸秆燃烧排放颗粒物的水平相当，玉米秸 秆排放水平高于小麦秸秆. 从燃烧模拟过程可以看出, 玉米秙秆燃烧时闷烧过程持续时间更长，而小麦秸秆明 火燃烧阶段更长，问烧阶段燃烧更不充分 ${ }^{[15]}$, 会排放更 多颗粒物.

表 $1 \quad \mathrm{PM}_{2.5}$ 及各组分排放因子 $(\mathrm{mg} / \mathrm{kg})^{a}$

Table 1 Emission factors of $\mathrm{PM}_{2.5}$ and the components $(\mathrm{mg} / \mathrm{kg})$

\begin{tabular}{llll}
\hline & 玉米秸秆 & 小麦秸秆(望都) & 小麦秸秆(郑州) \\
\hline $\mathrm{PM}_{2.5}$ & $1082.8 \pm 589.7$ & $835.7 \pm 471.3$ & $897.3 \pm 554.6$ \\
$\mathrm{OC}$ & $584.7 \pm 409.8$ & $392.0 \pm 266.6$ & $394.2 \pm 300.6$ \\
WSOC & $250.1 \pm 148.8$ & $145.2 \pm 101.0$ & $184.6 \pm 169.4$ \\
$\mathrm{EC}$ & $20.5 \pm 7.8$ & $21.5 \pm 5.4$ & $21.5 \pm 3.8$ \\
乙酸 & $1.7 \pm 1.4$ & $1.1 \pm 1.2$ & $1.3 \pm 1.7$ \\
甲酸 & $0.4 \pm 0.3$ & $0.4 \pm 0.5$ & $0.4 \pm 0.6$ \\
丁二酸 & $0.9 \pm 1.0$ & $0.8 \pm 1.2$ & $0.9 \pm 1.5$ \\
乙二酸 & $0.2 \pm 0.1$ & $0.3 \pm 0.2$ & $0.2 \pm 0.2$ \\
丙酸 & $\mathrm{N} . \mathrm{A}$. & $\mathrm{N} . \mathrm{A}$. & $\mathrm{N} . \mathrm{A}$. \\
甲磺酸 & $\mathrm{N} . \mathrm{A}$. & $\mathrm{N} . \mathrm{A}$. & $\mathrm{N} . \mathrm{A}$. \\
$\mathrm{K}^{+}$ & $30.4 \pm 21.5$ & $39.2 \pm 20.3$ & $51.9 \pm 23.2$ \\
$\mathrm{Cl}^{-}$ & $64.8 \pm 29.7$ & $50.8 \pm 14.1$ & $64.7 \pm 15.5$ \\
$\mathrm{NH}_{4}{ }^{+}$ & $11.2 \pm 10.1$ & $4.5 \pm 2.9$ & $4.6 \pm 2.0$ \\
$\mathrm{SO}_{4}{ }^{-}$ & $4.1 \pm 2.3$ & $4.7 \pm 2.4$ & $11.0 \pm 10.7$ \\
$\mathrm{NO}_{3}{ }^{-}$ & $0.4 \pm 0.3$ & $0.5 \pm 0.5$ & $1.0 \pm 1.4$ \\
$\mathrm{Na}^{+}$ & $0.1 \pm 0.1$ & $0.2 \pm 0.1$ & $2.7 \pm 4.1$ \\
$\mathrm{Mg}^{2+}$ & $0.01 \pm 0.02$ & N.A. & N.A. \\
$\mathrm{Ca}^{2+}$ & $0.02 \pm 0.03$ & N.A. & $0.01 \pm 0.02$ \\
\hline $\mathrm{NA}^{+}$ & &
\end{tabular}

${ }^{a}$ N.A.为未检出或结果低于仪器检测限.

运用本研究所得 $\mathrm{PM}_{2.5}$ 排放因子, 其中小麦秸秆排 放因子取两地平均值, 初步估算得到全国每年由玉米和 小麦秙秆露天燃烧产生 $\mathrm{PM}_{2.5}$ 的量分别为 63.0 和 29.7 Gg. 排放最多的 5 个省份分别是黑龙江、吉林、内蒙古、 山东、河南和河南、山东、河北、安徽、江苏, 这些省 份的排放对我国这两种秙秆燃烧排放总量的贡献分别 为 $54 \%$ 和 $76 \%$. 秸秆露天燃烧的污染物排放量与这些地 区农作物产量直接相关. 由于数据的缺乏, 本研究的估 
算未能考虑由于各省监管措施、燃烧条件等的差异对排 放量的影响. 虽然结果存在一定误差, 但这并不影响对 整体的参考意义. 在农作物种植面积较大的省份格外加 强收获季节秸秆露天燃烧的监管尤为重要.

\section{$2.2 \mathrm{PM}_{2.5}$ 化学组成及其排放因子}

秸秆燃烧排放 $\mathrm{PM}_{2.5}$ 主要化学组成的排放因子和比 例如表 1 和图 1 所示, 本研究检测到的组分共占颗粒物 总质量的 $73 \% \sim 82 \%$, 其中 $\mathrm{OM} / \mathrm{OC}$ 比值以 1.3 计 $^{[11]}$ 得到 有机物 $(\mathrm{OM})$ 的质量. 本研究及其它研究得到的不同种 类农作物秸秆燃烧排放 $\mathrm{PM}_{2.5}$ 主要组分的比例列于表 2 . $\mathrm{OC}$ 是秸秆燃烧排放 $\mathrm{PM}_{2.5}$ 中最主要的组分, 占总质量的 $31 \% \sim 51 \%$, 与之前研究 $26 \% \sim 69 \%$ 的结果一致 ${ }^{[11 \sim 13]}$. 两地小麦秸秆燃烧排放水平相当, 分别为 392.0 和 394.2 $\mathrm{mg} / \mathrm{kg}$; 玉米秸秆燃烧排放因子约为小麦秸秆的 1.5 倍. OC 中 $37 \% \sim 50 \%$ 为水溶性有机碳(WSOC), 略少于非水 溶性有机碳(WISOC), 与 Bae 等(2013)和 Guo 等(2014) 的研究结果相近 ${ }^{[22,23]}$. 水溶性有机碳(WSOC)同样呈现 玉米秸秆排放高于小麦秸秆的趋势. 乙酸是离子色谱可 以测定的 6 种低分子量有机酸中排放水平最高的组分, 平均为 $0.4 \sim 3.0 \mathrm{mg} / \mathrm{kg}$, 排放水平同样呈现玉米秸秆高 于小麦秸秆的趋势. $\mathrm{EC}$ 的排放因子远低于 $\mathrm{OC}$, 平均为 $16.1 \sim 26.6 \mathrm{mg} / \mathrm{kg}$, 占颗粒物总质量的 $1 \% \sim 4 \%$, 与之前 研究中 $1 \% \sim 23 \%$ 的结果一致(表 2). 玉米及两地小麦秸 秆 $\mathrm{EC}$ 排放水平相近.

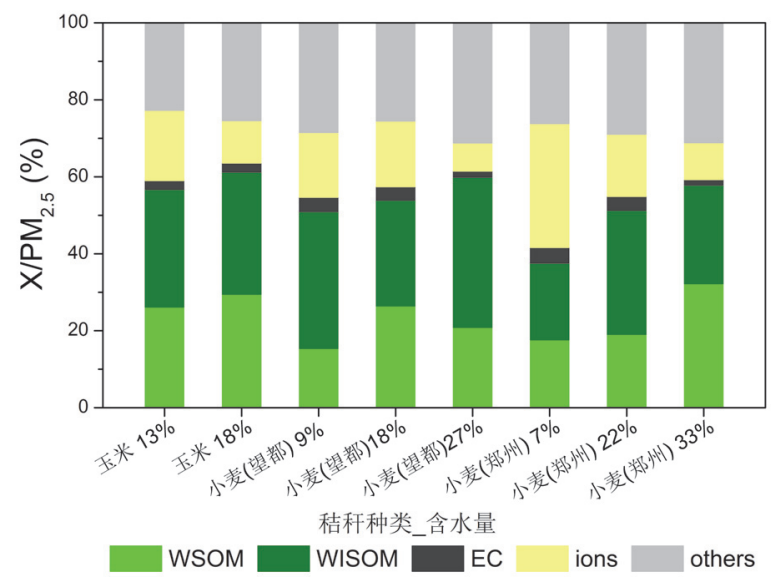

图 1 不同含水量秸秆燃烧排放 $\mathrm{PM}_{2.5}$ 化学组成

Figure 1 Chemical composition of $\mathrm{PM}_{2.5}$ emitted from the burning of straw with different moisture content

类似 $\mathrm{PM}_{2.5}$ 排放量的计算方法, 初步估算得到全国 每年由玉米和小麦秸秆露天燃烧产生 $\mathrm{OC}$ 的量分别为 34.0 和 $13.5 \mathrm{Gg}$; EC 分别为 1.2 和 $0.7 \mathrm{Gg}$. 我国每年由农 作物秸秆露天燃烧排放 OC、 EC 的量分别为 395.8 $402.9 \mathrm{Gg}$ 和 84.2 $100.0 \mathrm{Gg}^{[9,10]}$, 玉米和小麦这两种主要 粮食作物露天燃烧排放对我国秙秆露天燃烧排放 OC、 EC 总量的贡献分别为 $12 \%$ 和 $2 \%$. 但考虑到 $\mathrm{CaO}$ 等 ${ }^{[9]}$ 和 Yan 等 ${ }^{[10]}$ 的研究中所采用的排放因子大于本研究所得结
表 2 秸秆燃烧排放 $\mathrm{PM}_{2.5}$ 主要化学组成 $\left(\% \mathrm{PM}_{2.5}\right)$

Table 2 Chemical composition of $\mathrm{PM}_{2.5}$ emitted from straw burning $\left(\% \mathrm{PM}_{2.5}\right)$

\begin{tabular}{|c|c|c|c|c|c|}
\hline 秸秆种类 & $\mathrm{OC}$ & $\mathrm{EC}$ & $\mathrm{K}^{+}$ & $\mathrm{Cl}^{-}$ & 参考文献 \\
\hline \multirow{3}{*}{ 玉米秸秆 } & 34 & 3 & 9 & 23 & [11] \\
\hline & 33 & 14 & 14 & 26 & [13] \\
\hline & $47 \sim 51$ & 2 & $3 \sim 6$ & $7 \sim 10$ & 本研究 \\
\hline \multirow{3}{*}{ 小麦秸秆 } & 39 & 8 & 10 & 14 & [11] \\
\hline & 26 & 11 & 25 & 24 & [12] \\
\hline & 37 & 14 & 16 & 22 & [13] \\
\hline 小麦秸秆 (望都) & $42 \sim 50$ & $2 \sim 4$ & $2 \sim 7$ & $4 \sim 8$ & 本研究 \\
\hline 小麦秸秆 (郑州) & $31 \sim 48$ & $1 \sim 4$ & $3 \sim 14$ & $4 \sim 15$ & 本研究 \\
\hline 大麦秸秆 & 38 & 16 & 15 & 5 & [13] \\
\hline \multirow{2}{*}{ 水稻秸秆 } & 69 & 1 & 1 & 1 & [12] \\
\hline & 22 & 23 & 15 & 27 & [13] \\
\hline 甘蔗秸秆 & 37 & 15 & 12 & 18 & {$[13]$} \\
\hline
\end{tabular}

果, 两种秸秆露天燃烧排放对总量的贡献比例可能更 高.

水溶性无机离子组分共占颗粒物总质量的 $8 \%$ $32 \%, \mathrm{Cl}^{-}, \mathrm{K}^{+}$是其中浓度最高的组分, 其次是 $\mathrm{SO}_{4}{ }^{2-}$ 和 $\mathrm{NH}_{4}{ }^{+}$, 其它组分含量很低或未检出(表 1). $\mathrm{K}^{+}$一般认为 是生物质燃烧的示踪物，木材、秸秆等各类生物质燃烧 均会向大气中排放大量 $\mathrm{K}^{+[6,12 \sim 14]}$. 本研究得到 $\mathrm{K}^{+}$排放 因子为 $29.6 \sim 65.8 \mathrm{mg} / \mathrm{kg}$, 占 $\mathrm{PM}_{2.5}$ 总质量的 $2 \% \sim 14 \%$, 与之前研究中 $1 \% \sim 25 \%$ 的结果一致(表 2). 不同秸秆间 $\mathrm{K}^{+}$排放水平的相对高低不同于有机物，小麦秸秆的排 放水平整体上高于玉米秸秆，郑州小麦秸秆排放水平高 于望都小麦秸秆. 这可能与秸秆本身组成及燃烧过程火 焰温度的差异有关，燃烧温度较低时不利于 $\mathrm{K}^{+}$释放进 入烟气中. 这部分将在 2.3 节燃烧排放影响因素部分详 细分析. 本研究得到颗粒物中 $\mathrm{K}^{+} / \mathrm{EC}$ 比值为 $0.5 \sim 3.8$, 平均为 2.0 , 与其它研究中 $0.2 \sim 2.3$ 的结果一致 ${ }^{[6,11,12]}$. 除 $\mathrm{K}^{+}$外, 秸秆燃烧往往也会有高浓度的 $\mathrm{Cl}^{-}$排放, 本研 究得到 $\mathrm{Cl}^{-}$排放因子为 $48.7 \sim 75.8 \mathrm{mg} / \mathrm{kg}$, 占 $\mathrm{PM}_{2.5}$ 总质 量的 $4 \% \sim 15 \%$, 与之前研究中 $2 \% \sim 27 \%$ 的结果一致(表 2).

\section{3 颗粒物排放的影响因素}

本研究对玉米和小麦秙秆分别控制 2 个和 3 个含水 量梯度进行燃烧模拟，不同含水量梯度的秸秆燃烧排放 $\mathrm{PM}_{2.5}$ 的排放因子和组成如图 1 和图 2 所示. 随着秙秆含 水量的增加, $\mathrm{PM}_{2.5}$ 排放因子增加, 其中小麦秸秆中等含 水量和低等含水量相比变化不大，高等含水量排放显著 增加, 为低、中等含水量的 $2 \sim 3$ 倍; 颗粒物主要组分 OC 呈现同样的趋势, 且随含水量增加, 占颗粒物总质 量的比例也有所增加(图 1). 随秸秆含水量增加, 明火燃 烧阶段逐渐缩短，而闷烧阶段变长，问烧阶段秸秆燃烧 效率较低, 燃烧不充分会产生更多的颗粒物 ${ }^{[15,24]}$. 中等 
含水量小麦秸秆与低等含水量相比, $\mathrm{PM}_{2.5}$ 和 $\mathrm{OC}$ 的排放 因子没有明显增加趋势，这是由于低等含水量秸秆以明 火燃烧为主, 燃烧速度很快, 由于环境供氧量不足也可 能导致一定量的不完全燃烧, 颗粒物排放增加 ${ }^{[1]}$. 小麦 收获季节一般集中在 6 月, 太阳辐射强且环境温度较高, 秸秆中的水分在田间容易较快挥发, 实际燃烧状态大部 分较接近中等或低等含水量秸秆燃烧; 然而夏季降水较 多, 雨后秸秆含水量增加, 这种情况下对其进行露天燃 烧颗粒物排放量会显著增加. 玉米收获季节多集中在 10 月, 与夏季相比太阳辐射较弱而环境温度低, 秸秆中 水分很难较快挥发, 实际燃烧情况更接近中等或高等含 水量秸秆燃烧. 在秋收季节或雨后, 秸秆燃烧会排放更 多颗粒物, 尤其是碳质气溶胶, 对区域环境质量和周边 居民健康造成严重影响, 这时尤其要加强对秸秆露天燃 烧的监管力度. 随秸秆含水量的增加, EC 排放因子整体 上并没有明显变化(图 2); $\mathrm{EC}$ 占 $\mathrm{PM}_{2.5}$ 的比例呈现减小的 趋势(图 2), 这主要是由于随秸秆含水量增加, 颗粒物排 放量逐渐增加.

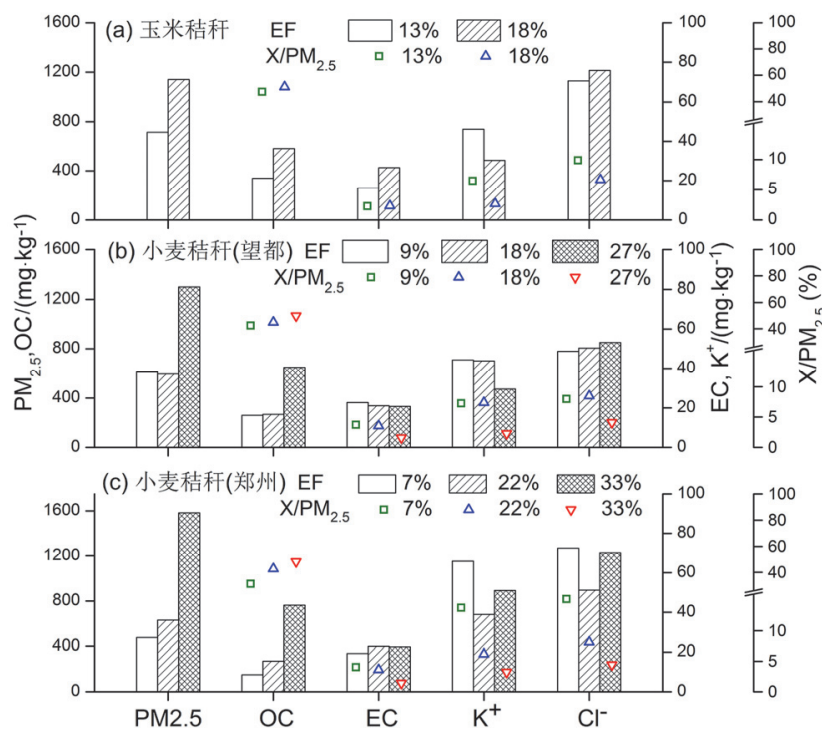

图 $2 \mathrm{PM}_{2.5}, \mathrm{OC}, \mathrm{EC}, \mathrm{K}^{+}, \mathrm{Cl}^{-}$排放因子及其占 $\mathrm{PM}_{2.5}$ 的比例

Figure 2 Emission factors of $\mathrm{PM}_{2.5}, \mathrm{OC}, \mathrm{EC}, \mathrm{K}^{+}, \mathrm{Cl}^{-}$and their contribution to $\mathrm{PM}_{2.5}$

随秸秆含水量的增加, 水溶性无机离子在颗粒物总 量中所占比例逐渐减小 (图 1). $\mathrm{K}^{+}$的排放因子整体呈现 减小的趋势, 其在颗粒物中所占比例随含水量增加而减 小的趋势更为明显(图 2); 其中玉米秸秆和郑州小麦秸 秆燃烧排放颗粒物中 $\mathrm{K}^{+}$所占比例随含水量增加逐渐降 低, 望都小麦秸秆高等含水量与中、低含水量相比明显 降低. $\mathrm{Cl}^{-}$排放因子随秸秆含水量增加没有明显变化趋 势, 但其在颗粒物中所占比例与 $\mathrm{K}^{+}$类似, 整体呈现出 随秸秆含水量增加而逐渐降低的趋势. 研究表明, 在生 物质燃烧热解过程中, $\mathrm{K}^{+}$和 $\mathrm{Cl}^{-}$由生物质燃料向烟气中 的释放受温度的影响. 虽然不同研究结果的具体温度值
存在一定差异, 但整体趋势均为燃烧达到一定温度以上 时, $\mathrm{K}^{+}$和 $\mathrm{Cl}^{-}$才开始从生物质燃料中释放进入烟气，随 温度升高释放进入烟气的比例增加 ${ }^{[25 ~ 27]}$. 本研究中 $\mathrm{K}^{+}$ 和 $\mathrm{Cl}^{-}$排放因子或在颗粒物中所占比例随秸秆含水量 增加而减小的趋势与燃烧过程火焰温度有关, 不同含水 量梯度秸秆燃烧过程火焰温度变化如图 3 所示. 秸秆燃 烧释放的热量一部分用于秙秆水分的蒸发, 另一部分使 燃烧温度升高. 随秸秆含水量增加水分蒸发所消耗的热 量逐渐增加，火焰所达到的温度降低，导致由生物质燃 料释放进入烟气的 $\mathrm{K}^{+}$和 $\mathrm{Cl}^{-}$比例逐渐减小. 因而，二者 在烟气中所占比例随含水量的增加, 即燃烧温度的降低 呈现减小趋势。望都小麦秸秆中等含水量与低等含水量 相比火焰温度没有明显变化(图 $3 \mathrm{~b}$ ), $\mathrm{K}^{+}$和 $\mathrm{Cl}^{-}$在颗粒物 中所占比例也没有明显降低趋势. 不同地区和季节温、 湿条件存在差异，即使是相当量的同种秸秆燃烧排放 $\mathrm{K}^{+}$的水平也可能存在差异，在同一区域内排除其它源 影响的情况下以 $\mathrm{K}^{+}$浓度表征生物质燃烧强度是可行的， 但差异较大的不同地区和季节间的比较需充分考虑燃 烧条件对排放的影响.



图 3 秸秆燃烧过程火焰温度变化

Figure 3 Temperature during the straw burning process

\section{3 结论}

本研究对我国最主要的两种粮食作物玉米和小麦 秸秆的燃烧排放进行模拟研究，玉米秸秆、望都小麦秸 秆和郑州小麦秙秆燃烧 $\mathrm{PM}_{2.5}$ 的排放因子分别为 1082.8 , 835.7 和 $897.3 \mathrm{mg} / \mathrm{kg}$. 玉米秸秆燃烧过程闷烧阶段更长, 燃烧不充分从而排放更多颗粒物. 估算得到全国每年由 
玉米和小麦秸秆露天燃烧排放 $\mathrm{PM}_{2.5}$ 的量分别为 63.0 和 $29.7 \mathrm{Gg}$, 在农作物种植面积较大的省份加强收获季节 秙秆露天燃烧的监管对大气污染控制尤为重要. OC 是 $\mathrm{PM}_{2.5}$ 中最主要的组分, 其中水溶性有机碳比例略小于 非水溶性有机碳. 玉米和小麦这两种主要粮食作物的秸 秆露天燃烧排放对我国秙秆露天燃烧排放 OC 总量的贡 献在 $12 \%$ 以上. $\mathrm{Cl}^{-}$和 $\mathrm{K}^{+}$是水溶性无机离子中浓度最高 的组分, 分别占 $\mathrm{PM}_{2.5}$ 总质量的 $4 \% \sim 15 \%$ 和 $2 \% \sim 14 \%$, $\mathrm{K}^{+} / \mathrm{EC}$ 值为 $0.5 \sim 3.8$. 各物种在 $\mathrm{PM}_{2.5}$ 中所占比例与之前 秸秆燃烧排放颗粒物的研究结果一致.

秸秆含水量是影响秸秆燃烧排放颗粒物水平和特 征的重要因素. 随秙秆含水量的增加, 问烧阶段变长, 明火燃烧阶段缩短, 不充分燃烧使 $\mathrm{PM}_{2.5}$ 和 $\mathrm{OC}$ 排放因 子增加, 高含水量小麦秸秆排放是中、低含水量排放的 2 3 倍. 随秸秆含水量增加, 燃烧产生的热量更多的被 秸秆中的水分蒸发消耗, 燃烧火焰温度降低, 由生物质 燃料释放进入烟气中的 $\mathrm{K}^{+}$和 $\mathrm{Cl}^{-}$比例减小, 二者在燃烧 排放颗粒物中的比例因此降低. 以 $\mathrm{K}^{+}$作为示踪物表征 不同地区和季节的生物质燃烧强度时应充分考虑燃烧 条件不同对其排放产生的影响. 在秋收季节或雨后, 尤 其要加强对秸秆露天燃烧的监管力度.

\section{4 实验部分}

\section{1 模拟系统及可靠性验证}

本研究所采用的生物质燃烧模拟系统主要包括燃 烧系统、稀释系统、动力系统及采样与数据记录系统 4 部分, 所有管路和通道均由不锈钢材料制成以有效减少 颗粒物在管壁的吸附损失，系统结构图见 $\mathrm{He}$ 等 (2010) ${ }^{[28]}$. 基本的燃烧模拟过程为: (1)将秸秆在不锈钢 燃烧平台上点燃, 燃烧产生的颗粒物被上方烟气罩收 集，在负压作用下由等速采样口进入稀释系统. 燃烧过 程由温度探头实时监测并记录火焰温度变化. (2)稀释系 统前端管路缠有加热带, 减少由于烟气在与干空气混合 稀释过程中温度骤降引起在管壁沉积损失. 在此, 烟气 首先被 $70 \mathrm{~L} / \mathrm{min}$ 的干空气一级稀释, 混合气经“文丘里” 变速后被 $20 \mathrm{~L} / \mathrm{min}$ 的干空气二级稀释, 干空气流量均由 流量计准确控制, 可实现对烟气进行固定比例的稀释. 本实验设计的烟气稀释倍数为 10 倍. (3)混合气在烟气 停留室内混合后被采样, 采样系统共分 6 路, 各路流速 均为 $16.7 \mathrm{~L} / \mathrm{min}$; 其中 $1 \sim 5$ 路装有 $\mathrm{PM}_{2.5}$ 旋风式切割头, 用于离线膜采样, 并由 3 个外置泵提供采样动力, 第 6 路用于连接其它离线和在线仪器采样与分析, 其中包括 二氧化碳分析仪(Thermo, Modle 410i) 实时测定 $\mathrm{CO}_{2}$ 浓 度.

为保证模拟系统的可靠性, 在实验开始前对其稀释 比和各采样通道间的平行性进行测试: (1)系统稀释比测 定: 由烟气罩向系统持续注入稳定浓度的 $\mathrm{CO}_{2}$
(99.99\%), 待系统稳定后用二氧化碳分析仪分别测定稀 释前后 $\mathrm{CO}_{2}$ 的浓度, 分别将稀释前后浓度的测定值减去 对应本底浓度作为稀释前后注入 $\mathrm{CO}_{2}$ 的实际浓度, 两者 相除即为系统实际稀释比. 实际测定值为 10.6 , 可以实 现设定的对烟气进行稳定 10 倍稀释. (2)各采样通道间 的平行性测定：为保证采样过程中各通道间的一致性， 按照 4.2 和 4.3 节部分介绍的实际实验过程对玉米秸秆 的燃烧进行模拟和采样, 测定采集到的石英膜样品的有 机碳(OC)和元素碳(EC). 预实验结果表明, 各通道采样 膜上的 $\mathrm{OC}$ 为 $99.4 \sim 101.7 \mu \mathrm{g} / \mathrm{cm}^{2}$, EC 为 $2.4 \sim 3.1 \mu \mathrm{g} / \mathrm{cm}^{2}$, 偏差在 $5 \%$ 以内，各采样通道间具有很好的一致性.

\section{2 样品采集}

根据 2014 年统计年鉴, 2004 2013 年我国小麦和 玉米秸秆的种植面积分别占粮食作物总种植面积的 $22 \%$ 和 $29 \%$, 产量分别占粮食作物总产量的 $21 \%$ 和 $32 \%$. 本研究选取小麦和玉米这两种我国最主要的粮食作物, 对其秸秆燃烧进行模拟. 2014 年 6 月，小麦收获季节在 河南省郑州及河北省望都收集小麦秸秆；10 月，玉米收 获季节在河南省邓县收集玉米秸秆.

根据欧盟标准(CEN/TS 14774-2004)中的固态生物 质燃料水分测定方法，将一定质量秸秆在 $105{ }^{\circ} \mathrm{C}$ 的烘 箱内烘干至恒重，测定前后的质量变化计算其含水量. 测定结果显示，所收集玉米秸秆、郑州小麦秸秆和望都 小麦秙秆的初始含水量分别为 $13 \%, 7 \%$ 和 $9 \%$. 实验过 程中, 燃烧模拟所需秸秆均在实验室内准确控制含水 量: 按一定质量比在塑料箱内逐层平铺秸秆并喷洒超纯 水(Milli-Q Gradient 纯水机, $18.2 \mathrm{M} \Omega \bullet \mathrm{cm}$ ), 至少 $20 \mathrm{~h}$ 后 重新称重并计算实际含水量. 3 种秸秆燃烧模拟实验的 基本信息和参数见表 3 .

在 5 路离线膜采样中, 第 3 路采集 Teflon 膜 (Whatman Inc., USA, $47 \mathrm{~mm}$ ), 1, 2, 4, 5 路采集石英纤维 滤膜(Whatman, UK, $47 \mathrm{~mm}$ ). 每次实验均为将生物质燃 料点火后开始采样, 火焰熄灭且 $\mathrm{CO}_{2}$ 降低至燃烧前浓度 水平后停止采样，每次采样持续 20 35 min. 为保证实 验结果的可靠性, 对每种秸秆每个含水量梯度的燃烧均 进行 3 次模拟. 用干空气将模拟系统管路冲洗干净后, 在没有秙秆燃烧的情况下，按照同样过程采集样品，作 为现场空白样品.

\section{3 分析方法}

采样前后将 Teflon 膜在恒温恒湿 $\left[(20 \pm 1){ }^{\circ} \mathrm{C},(40 \pm\right.$ $5) \%$ ] 超净室平衡 $24 \mathrm{~h}$ 后称量, 根据采样前后的质量差得 到采集 $\mathrm{PM}_{2.5}$ 的质量. 将称量完毕的 Teflon 膜用 $10 \mathrm{~mL}$ 去离子水(Milli-Q Gradient 纯水机, $18.2 \mathrm{M} \Omega \bullet \mathrm{cm}$ )超声提 取 $30 \mathrm{~min}$, 经 $0.45 \mu \mathrm{m}$ PTFE 滤膜(Gelman Sciences)过滤 后运用离子色谱法(Dionex ICS-2000/2500)测定水溶性 离子组分 $\left(\mathrm{Na}^{+}, \mathrm{NH}_{4}{ }^{+}, \mathrm{K}^{+}, \mathrm{Mg}^{2+}, \mathrm{Ca}^{2+}, \mathrm{Cl}^{-}, \mathrm{NO}_{3}{ }^{-}\right.$, $\mathrm{SO}_{4}{ }^{2-}$ ) 和低分子量有机酸(乙酸、丙酸、甲酸、甲磺酸、 
表 3 秸秆燃烧模拟的实验参数

Table 3 Parameters of straw burning simulation

\begin{tabular}{llllll}
\hline 秸秆种类 & 收集地点/时间 & 含水量 $/ \%$ & 燃料质量 $/ \mathrm{kg}$ & 燃烧持续时间 $/ \mathrm{min}$ & 模拟次数 \\
\hline \multirow{2}{*}{ 玉米秸秆 } & 河南邓县 $/ 2014.10$ & 13 & $1.6 \sim 1.9$ & $30 \sim 35$ & 3 \\
& & 18 & $1.8 \sim 1.9$ & $30 \sim 35$ & 3 \\
& & 9 & $0.8 \sim 0.9$ & $23 \sim 25$ & 3 \\
小麦秸秆 & 河北望都/2014.06 & 18 & $1.0 \sim 1.1$ & $24 \sim 26$ & 3 \\
& & 27 & $0.8 \sim 1.2$ & $20 \sim 30$ & 3 \\
& & 7 & $1.0 \sim 1.1$ & $20 \sim 25$ & 3 \\
小麦秸秆 & 河南郑州/2014.06 & 22 & $0.9 \sim 1.0$ & $24 \sim 26$ & 3 \\
& & 33 & $0.7 \sim 0.8$ & $25 \sim 30$ & 3 \\
\hline
\end{tabular}

丁二酸、乙二酸 ${ }^{29]}$. 运用美国 Sunset EC/OC 分析仪热光 法分析石英纤维滤膜采集颗粒物中的有机碳(OC)和元 素碳 $(\mathrm{EC})^{[30]}$, 运用岛津水溶性有机碳分析仪 (TOC-5000A)分析水溶性有机碳(WSOC).

烟气中各组分的排放因子由下式计算得到, 单位以 $\mathrm{mg} / \mathrm{kg}$ 干重燃料计:

$$
E F_{i}=\frac{\delta m_{i}}{\delta M_{\mathrm{dry}}} \times \frac{Q_{0}}{Q_{\text {sample }}}=\frac{\delta m_{i}}{\delta M_{\mathrm{dry}}} \times \frac{S_{0}}{S_{\text {sample }}}
$$

其中, $E F_{i}$ 表示物种 $i$ 的排放因子; $\delta m_{i}$ 表示采集的 $\mathrm{PM}_{2.5}$ 中物种 $i$ 的总质量; $\delta M_{\mathrm{dry}}$ 表示秥秆干重; $Q_{0}$ 表示燃烧产 生烟气的总量; $Q_{\text {sample }}$ 表示采集到的未稀释烟气的量; $S_{0}$ 表示烟气通道的横截面积; $S_{\text {sample }}$ 表示采样口面积. 由于 烟气为等速采样, 烟气总量与采样量的比值等于烟气通 道与采样口面积的比值.

在得到排放因子的基础上，运用下式估算某一地区 玉米或小麦秸秆燃烧排放颗粒态污染物的总量:

$$
M_{i j}=P_{i} \times R P R_{i} \times\left(1-M C_{i}\right) \times B_{i} \times F_{i} \times E F_{i j}
$$

其中, $M_{i j}$ 表示 $i$ 秥秆燃烧产生的物种 $j$ 的排放量, $P_{i}$ 表示 $i$ 农作物的产量, $R P R_{i}$ (Residue-to-Product Ratio)表示 $i$ 农 作物秸秆与其产量的比值, $M C_{i}$ (Moisture Content)表示 $i$ 秸秆的含水量, $B_{i}$ 表示 $i$ 秸秆露天燃烧的量占秸秆总量 的比例, $F_{i}$ 表示 $i$ 秸秆的燃烧效率, $E F_{i j}$ 表示 $i$ 秸秆燃烧产 生的物种 $j$ 的排放因子. 本研究采用 2014 年统计年鉴公 布的 2013 年我国小麦和玉米产量数据; $R P R_{i}$ 参考 Koopmans 等(1997)的研究结果, 玉米和小麦均采用 $2.0^{[31]} ; M C_{i}$ 采用本研究测得的秥秆的实际初始含水量, 即玉米秸秆为 $13 \%$, 小麦秸秆取两地平均值 $(8 \%) ; B_{i}$ 采 用 $17 \%{ }^{[32]}$; 参考 Turn 等(1997)和 Streets 等(2003)的研究, 燃烧效率 $F_{i}$ 取 $90 \%{ }^{[8,13]} ; E F_{i j}$ 即为本研究得到的排放因 子.

\section{致谢}

感谢张宜升老师在燃烧模拟实验中的帮助和指导, 感谢郭松老师在数据分析及论文写作方面的指导, 感谢 李建峰提供的田间秸秆燃烧照片.

\section{References}

[1] Simoneit, B. R. T. Appl. Geochem. 2002, 17, 129.

[2] Anderson, B. E.; Grant, W. B.; Gregory, G. L.; Browell, E. V.; Collins, J. E.; Sachse, G. W.; Bagwell, D. R.; Hudgins, C. H.; Blake, D. R.; Blake, N. J. J. Geophys. Res. 1996, 101, 24117.

[3] Cheng, Y.; Engling, G.; He, K. B.; Duan, F. K.; Ma, Y. L.; Du, Z. Y.; Liu, J. M.; Zheng, M.; Weber, R. J. Atmos. Chem. Phys. 2013, 13, 7765 .

[4] Khan, A. A.; de Jong, W.; Jansens, P. J.; Spliethoff, H. Fuel Process. Technol. 2009, 90, 21.

[5] Radzi bin Abas, M.; Oros, D. R.; Simoneit, B. R. Chemosphere 2004, 55, 1089.

[6] Andreae, M. O.; Merlet, P. Global Biogeochem. Cycles 2001, 15, 955.

[7] Iinuma, Y.; Brüggemann, E.; Gnauk, T.; Müller, K.; Andreae, M. O.; Helas, G.; Parmar, R.; Herrmann, H. J. Geophys. Res. 2007, 112.

[8] Streets, D. G.; Yarber, K. F.; Woo, J. H.; Carmichael, G. R. Global Biogeochem. Cycles 2003, 17, 1099.

[9] Cao, G. L.; Zhang, X. Y.; Zheng, F. C. Atmos. Environ. 2006, 40, 6516.

[10] Yan, X. Y.; Ohara, T.; Akimoto, H. Atmos. Environ. 2006, 40, 5262.

[11] Li, X.; Wang, S.; Duan, L.; Hao, J.; Li, C.; Chen, Y.; Yang, L. Environ. Sci. Technol. 2007, 41, 6052.

[12] Hays, M. D.; Fine, P. M.; Geron, C. D.; Kleeman, M. J.; Gullett, B. K. Atmos. Environ. 2005, 39, 6747.

[13] Turn, S. Q.; Jenkins, B. M.; Chow, J. C.; Pritchett, L. C.; Campbell, D.; Cahill, T.; Whalen, S. A. J. Geophys. Res.-Atmos. 1997, 102, 3683.

[14] Li, J. F.; Song, Y.; Mao, Y.; Mao, Z. C.; Wu, Y. S.; Li, M. M.; Huang, X.; He, Q. C.; Hu, M. Atmos. Environ. 2014, 92, 442.

[15] Sanchis, E.; Ferrer, M.; Calvet, S.; Coscollà, C.; Yusà, V.; Cambra-López, M. Atmos. Environ. 2014, 98, 25.

[16] Shen, G.; Xue, M.; Wei, S.; Chen, Y.; Wang, B.; Wang, R.; Shen, H.; Li, W.; Zhang, Y.; Huang, Y.; Chen, H.; Wei, W.; Zhao, Q.; Li, B.; Wu, H.; Tao, S. J. Environ. Sci. 2013, 25, 511.

[17] Lu, H.; Zhu, L.; Zhu, N. Atmos. Environ. 2009, 43, 978.

[18] Ortiz de Zárate, I.; Ezcurra, A.; Lacaux, J. P.; Van Dinh, P. Atmos. Environ. 2000, 34, 3183.

[19] Nguyen, B. C.; Putaud, J. P.; Mihalopoulos, N.; Bonsang, B.; Doan, C. Environ. Monit. Assess. 1994, 31, 131.

[20] Levin, E. J. T.; McMeeking, G. R.; Carrico, C. M.; Mack, L. E.; Kreidenweis, S. M.; Wold, C. E.; Moosmüller, H.; Arnott, W. P.; Hao, W. M.; Collett, J. L.; Malm, W. C. J. Geophys. Res. 2010, 115.

[21] Dhammapala, R.; Claiborn, C.; Corkill, J.; Gullett, B. Atmos. Environ. 2006, 40, 1007.

[22] Guo, S.; Hu, M.; Guo, Q.; Shang, D. Acta Chim. Sinica 2014, 72, 658. (郭松，胡敏，郭庆丰，尚冬杰，化学学报, 2014, 72, 658.)

[23] Bae, M.-S.; Park, S.-S. Asian J. Atmos. Environ. 2013, 7, 95.

[24] Chen, L. W. A.; Verburg, P.; Shackelford, A.; Zhu, D.; Susfalk, R.; Chow, J. C.; Watson, J. G. Atmos. Chem. Phys. 2010, 10, 6617.

[25] Bjorkman, E.; Stromberg, B. Energy Fuels 1997, 11, 1026.

[26] Jensen, P. A.; Frandsen, F. J.; Dam-Johansen, K.; Sander, B. Energy Fuels 2000, 14, 1280.

[27] Knudsen, J. N.; Jensen, P. A.; Dam-Johansen, K. Energy Fuels 2004, 18,1385 . 
[28] He, L. Y.; Lin, Y.; Huang, X. F.; Guo, S.; Xue, L.; Su, Q.; Hu, M.; Luan, S. J.; Zhang, Y. H. Atmos. Chem. Phys. 2010, 10, 11535.

[29] Guo, S.; Hu, M.; Wang, Z. B.; Slanina, J.; Zhao, Y. L. Atmos. Chem. Phys. 2010, 10, 947

[30] Huan, N.; Zeng, L. M.; Shao, M.; Cui, L.; Mao, J. T.; Zhou, N.; Dong, H. B.; Yu, Z. Y.; Luo, Z. M. Acta Sci. Nat. Univ. Pekinensis
2006, 42, 265 (in Chinese). (狍宁，曾立民，邵敏，崔良，毛节泰， 周楠, 董华斌, 俞仲英, 罗志明, 北京大学学报(自然科学版), 2006, 42, 265.)

[31] Koopmans, A.; Koppejan, J. Regional Consultation on Modern Applications of Biomass Energy 1997, 6 .

[32] Hao, W. M.; Liu, M. H. Global Biogeochem. Cycles 1994, 8, 495.

(Lu, Y.) 